\title{
A dynamic IS-LM model with adaptive expectations
}

\author{
Moisă Altăr \\ Academy of Economic Studies- Bucharest
}

\begin{abstract}
We analyze the stability of a discrete-time dynamic model with an IS-ML structure. We assume that the Aggregate Supply function is of Lucas type and the monetary policy rule is of Friedman type. The mechanism of expectations formation is assumed to be of adaptive type (Friedman-Cagan).

In its final form, the model contains two state-variables, namely money supply and expected inflation. From the mathematical point of view, it is an affine discrete-time system, whose stability properties are analyzed in the paper.

We deduce sufficient conditions concerning the „learning coefficient” involved in the Friedman-Cagan type of forecast equation, so that the model be stable.
\end{abstract}

\section{The model}

We assume that the functions IS and LM are of Cobb-Douglas type :

$$
\begin{aligned}
& \text { IS: } Y=A \cdot Y^{c} \cdot e^{-g \cdot r} \\
& \text { LM: } \frac{M}{P}=Y^{l_{1}} \cdot e^{-l_{2}\left(r+\pi^{e}\right)}
\end{aligned}
$$

The variables are the following :

- $Y$-GDP size;

- $M-$ nominal money supply;

- $P$ - price index ;

- $r$ - real interest rate;

- $\pi^{e}$ - expected inflation rate;

- $A, c, g, l_{1}, l_{2}$ - parameters

In logarithmic form, equations(1) şi (2) become:

$$
\begin{gathered}
y=a+c \cdot y-g \cdot r \\
m=l_{1} \cdot y-l_{2}\left(r+\pi^{e}\right)
\end{gathered}
$$


We used the following notations:

$$
y=\ln (Y), m=\ln \left(\frac{M}{P}\right), a=\ln (A)
$$

The coordinates of the equilibrium point are:

$$
\left\{\begin{array}{l}
y^{*}=\frac{1}{1-c+g \cdot \frac{l_{1}}{l_{2}}}\left(a+\frac{g}{l_{2}} m+g \cdot \pi^{e}\right) \\
r^{*}=\frac{1}{l_{2}}\left(l_{1} \cdot y^{*}-m-l_{2} \cdot \pi^{e}\right)
\end{array}\right.
$$

We denote by $k$ the multiplyer :

$$
k=\frac{1}{1-c+g \cdot \frac{l_{1}}{l_{2}}}
$$

The first relationship in (6) becomes :

$$
y^{*}=k \cdot\left(a+\frac{g}{l_{2}} m+g \cdot \pi^{e}\right)
$$

Relation (8) expresses the fact that GDP (in logarithmic form) depends on the money supply $(\mathrm{m})$, on the expected inflationi $\left(\pi^{\mathrm{e}}\right)$, as well as on the coefficient ,a”, which reflects fiscal policy and the level of net export.

We assume that the quantity of money supplied by the Central Bank is governed by a Friedman type monetary policy rule:

$$
\begin{aligned}
& M_{t+1}=M_{t} \cdot e^{\mu} ; \mu=\text { constant } \\
& \quad \text { or } \\
& \ln \left(\frac{M_{t+1}}{M_{t}}\right)=\mu
\end{aligned}
$$

We assume that the aggregative supply (AS) is modeled by the following Phillips - Lucas type function:

$$
y_{t}=\bar{y}+\frac{1}{\theta}\left(\pi_{t}-\pi_{t}^{e}\right)
$$

where $\bar{y}$ represents the ,natural” size of GDP (in logarithmic form), and $\pi_{t}$ - the size of inflation. The coefficient $\theta$ is given. From (11) it follows :

$$
\pi_{t}=\pi_{t}^{e}+\theta \cdot\left(y_{t}-\bar{y}\right)
$$

As concerns inflation $\pi_{t}$, it is defined by : 


$$
P_{t+1}=P_{t} \cdot e^{\pi_{t}}
$$

or :

$$
\pi_{t}=\ln \left(\frac{P_{t+1}}{P_{t}}\right)
$$

As concerns the mechanism by which economic agents form expectations of future inflation, we assume that it is of adaptive type (Friedman - Cagan): $\pi^{e}{ }_{t+1}=\pi_{t}^{e}+\delta\left(\pi_{t}-\pi_{t}^{e}\right), 0 \leq \delta \leq 1$

The learning parameter $\delta$ is given.

Using relation (12), relation (15) becomes:

$$
\pi^{e}{ }_{t+1}=\pi_{t}^{e}+\delta \cdot \theta\left(y_{t}-\bar{y}\right)
$$

Using relation (8):

$$
y_{t}=y_{t}{ }^{*}=k \cdot\left(a+\frac{g}{l_{2}} m_{t}+g \cdot \pi_{t}^{e}\right)
$$

the dynamic equation (16) for expected inflation becomes:

$$
\begin{aligned}
& \pi_{t+1}^{e}=\pi_{t}^{e}+\delta \cdot \theta\left[k \cdot\left(a+\frac{g}{l_{2}} m_{t}+g \cdot \pi_{t}^{e}\right)-\bar{y}\right], \quad \text { or: } \\
& \pi^{e}{ }_{t+1}=\delta \cdot \theta \cdot k \cdot \frac{g}{l_{2}} m_{t}+(1+\delta \cdot \theta \cdot k \cdot g) \pi_{t}^{e}+\delta \cdot \theta \cdot(k \cdot a-\bar{y})
\end{aligned}
$$

We denote:

$\alpha=\theta \cdot k \cdot \frac{g}{l_{2}} ; h=\theta \cdot(k \cdot a-\bar{y})$

The dynamic equation (18) becomes:

$$
\pi^{e}{ }_{t+1}=\delta \cdot \alpha m_{t}+\left(1+\delta \cdot \alpha \cdot l_{2}\right) \pi_{t}^{e}+\delta \cdot h
$$

As concerns money supply, we have :

$$
m_{t+1}-m_{t}=\ln \frac{M_{t+1}}{P_{t+1}}-\ln \frac{M_{t}}{P_{t}}=\ln \frac{M_{t+1}}{M_{t}}-\ln \frac{P_{t+1}}{P_{t}}
$$

Using monetary policy rule (10), as well as the definition of inflation (14), we obtain:

$$
m_{t+1}-m_{t}=\mu-\pi_{t} \text { or } m_{t+1}=m_{t}+\mu-\pi_{t}
$$

Using relations (12) and (17), it follows: 


$$
\begin{gathered}
m_{t+1}=m_{t}-\pi_{t}^{e}-\theta \cdot\left[k \cdot\left(a+\frac{g}{l_{2}} m_{t}+g \cdot \pi_{t}^{e}\right)-\bar{y}\right]+\mu \quad \text { or } \\
m_{t+1}=\left(1-\theta \cdot k \cdot \frac{g}{l_{2}}\right) m_{t}-(1+\theta \cdot k \cdot g) \pi_{t}^{e}-\theta \cdot[k \cdot a-\bar{y}]+\mu .
\end{gathered}
$$

Using notations (19), we obtain:

$$
m_{t+1}=(1-\alpha) m_{t}-\left(1+\alpha \cdot l_{2}\right) \pi_{t}^{e}+\mu-h
$$

The dynamics of the system (the dynamicIS-LM model), in the variant proposed on the basis of the adopted assumptions, is given by relations (20) and (22).

$$
\left\{\begin{array}{l}
m_{t+1}=(1-\alpha) m_{t}-\left(1+\alpha \cdot l_{2}\right) \pi_{t}^{e}+\mu-h \\
\pi^{e}{ }_{t+1}=\delta \cdot \alpha m_{t}+\left(1+\delta \cdot \alpha \cdot l_{2}\right) \pi_{t}^{e}+\delta \cdot h
\end{array}\right.
$$

\section{Analysis of the dynamics}

In order to analyze the dynamics of system (23), we shall calculate the stationary trajectories :

$$
\begin{gathered}
m_{t+1}=m_{t}=m=\text { constant, } \forall t \in N \\
\pi^{e}{ }_{t+1}=\pi_{t}^{e}=\pi^{e}=\text { constant, } \forall t \in N \\
m=-\frac{1+\alpha \cdot l_{2}}{\alpha} \pi^{e}+\frac{1}{\alpha}(\mu-h) \text { for }\left(\mathrm{m}_{\mathrm{t}+1}=m_{t}=m\right) \\
m=-l_{2} \cdot \pi^{e}-\frac{1}{\alpha} h \text { for }\left(\pi^{\mathrm{e}}{ }_{t+1}=\pi_{t}{ }^{e}=\pi^{e}\right)
\end{gathered}
$$

The equilibrium (steady-state) point, i.e. the solution of the system formed of equations (23) and (24), is :

$$
\left\{\begin{array}{l}
\hat{\pi}^{e}=\mu \\
\hat{m}=-l_{2} \cdot \mu-\frac{1}{\alpha} h
\end{array}\right.
$$

Taking into account notations (19), it follows :

$$
\left\{\begin{array}{l}
\hat{\pi}^{e}=\mu \\
\hat{m}=\frac{l_{2}}{g}\left(\frac{1}{k} \bar{y}-a-g \cdot \mu\right)
\end{array}\right.
$$

Using relations (28), we obtain from (8) the equilibrium value for the GDP :

$$
\hat{Y}^{*}=\bar{Y}
$$


Relation (29) shows that, in equilibrium, the size of GDP will coincide with its ,natural" value.

At the same time, the first relation in (28) shows that, at equilibrium, expected inflation coincides with the growth rate of money supply.

To check the stability of the dynamic IS-LM system, we shall solve the system (23). This is an affine system of difference equations. Taking into account (27), it is easy to prove that system (23) can be rewritten as:

$$
\left\{\begin{array}{l}
m_{t+1}-\hat{m}=(1-\alpha)\left(m_{t}-\hat{m}\right)-\left(1+\alpha \cdot l_{2}\right)\left(\pi_{t}^{e}-\pi^{e}\right) \\
\pi^{e}{ }_{t+1}-\pi^{e}=\delta \cdot \alpha\left(m_{t}-\hat{m}\right)+\left(1+\delta \cdot \alpha \cdot l_{2}\right)\left(\pi_{t}^{e}-\hat{\pi}\right)
\end{array}\right.
$$

We denote:

$$
\left\{\begin{array}{l}
m_{t}-\hat{m}=u_{t} \\
\pi_{t}^{e}-\pi^{e}=z_{t}
\end{array}\right.
$$

The variables $u_{t}$ and $z_{t}$ represent the deviations from the respective equilibrium values.

With notations (31), system (30) can be written as :

$$
\left\{\begin{array}{l}
u_{t+1}=(1-\alpha) \cdot u_{t}-\left(1+\alpha \cdot l_{2}\right) \cdot z_{t} \\
z_{t+1}=\delta \cdot \alpha \cdot u_{t}+\left(1+\delta \cdot \alpha \cdot l_{2}\right) \cdot z_{t}
\end{array}\right.
$$

The difference equations system (32) has the advantage of being linear. In order to write system (32) in matrix form, we denote:

$$
X_{t}=\left(\begin{array}{l}
u_{t} \\
z_{t}
\end{array}\right) ; \mathrm{M}=\left(\begin{array}{cc}
1-\alpha & -\left(1+\alpha \cdot 1_{2}\right) \\
\delta \cdot \alpha & 1+\delta \cdot \alpha \cdot 1_{2}
\end{array}\right)
$$

System (32) becomes:

$$
X_{t+1}=M \cdot X_{t}
$$

From (34), we obtain, successively:

$X_{1}=M \cdot X_{0} ; \ldots X_{n}=M^{n} \cdot X_{0}$

Hence, the general solution of equation (34) is:

$$
X_{t}=M^{t} \cdot X_{0}
$$

It is more convenient to represent matrix $M$ in Jordan form:

$$
M=K \cdot \Lambda \cdot K^{-1}
$$

where $\Lambda$ is the diagonal matrix:

$$
\Lambda=\left(\begin{array}{cc}
\lambda_{1} & 0 \\
0 & \lambda_{2}
\end{array}\right)
$$


$\lambda_{1}$ and $\lambda_{2}$ being the eigenvalues of matrix $M$, and $K$ is the matrix whose columns are the eigenvectors of matrix $M^{1}$.

The characteristic equation of matrix $M$ is:

$\operatorname{det}\left(M-\lambda \cdot I_{2}\right)=0$

i.e:

We obtain:

$$
\left|\begin{array}{cc}
1-\alpha-\lambda & -\left(1+\alpha \cdot l_{2}\right) \\
\delta \cdot \alpha & 1+\delta \cdot \alpha \cdot l_{2}-\lambda
\end{array}\right|=0
$$

$$
\lambda^{2}-\left(1-\alpha+1+\delta \cdot \alpha \cdot l_{2}\right) \lambda+\delta \cdot \alpha\left(1+\alpha \cdot l_{2}\right)+(1-\alpha)\left(1+\delta \cdot \alpha \cdot l_{2}\right)=0
$$

or:

$$
\lambda^{2}-\left(2-\alpha+\delta \cdot \alpha \cdot l_{2}\right) \lambda+1-\alpha+\delta \cdot \alpha+\delta \cdot \alpha \cdot l_{2}=0
$$

Substituting the solutions $\lambda_{1}$ and $\lambda_{2}$ of ecuation (39) and taking into account (36), the solution (35) of system (34) becomes:

$$
X_{t}=\left[K \cdot \Lambda \cdot K^{-1}\right]^{t} \cdot X_{0}
$$

It is easy to see that:

$$
\left[K \cdot \Lambda \cdot K^{-1}\right]^{t}=K \cdot \Lambda^{t} \cdot K^{-1}
$$

In fact, the above relationship represents one of the advantages of the Jordan decomposition of a matrix.Taking into account that:

$$
\Lambda^{t}=\left[\begin{array}{cc}
\lambda_{1} & 0 \\
0 & \lambda_{2}
\end{array}\right]^{\cdot t}=\left[\begin{array}{cc}
\lambda_{1}{ }^{t} & 0 \\
0 & \lambda_{2}{ }^{t}
\end{array}\right]
$$

solution (40) becomes:

$$
X_{t}=K \cdot\left[\begin{array}{cc}
\lambda_{1}^{t} & 0 \\
0 & \lambda_{2}{ }^{t}
\end{array}\right] \cdot K^{-1} \cdot X_{0}
$$

We denote:

$$
\left(\begin{array}{l}
m_{t} \\
\pi_{t}^{e}
\end{array}\right)=\Psi_{t}
$$

Taking into account (31) and the first relation in (33), it follows:

$$
\Psi_{t}=\hat{\Psi}+X_{t}
$$

Obviously, the equilibrium solution is:

$$
\hat{\Psi}=\left(\begin{array}{c}
\hat{m} \\
\hat{\pi}^{e}
\end{array}\right)
$$

\footnotetext{
${ }^{1}$ See the mathematical note at the end of the section
} 
Taking into account (41), solution (43) can be written as:

$$
\begin{array}{r}
\left(\begin{array}{l}
m_{t} \\
\pi_{t}^{e}
\end{array}\right)=\left(\begin{array}{c}
\hat{m} \\
\hat{\pi}^{e}
\end{array}\right)+K \cdot\left[\begin{array}{cc}
\lambda_{1}^{t} & 0 \\
0 & \lambda_{2}{ }^{t}
\end{array}\right] \cdot K^{-1} \cdot X_{0} \text { or } \\
\left(\begin{array}{l}
m_{t} \\
\pi_{t}^{e}
\end{array}\right)=\left(\begin{array}{l}
\hat{m} \\
\hat{\pi}^{e}
\end{array}\right)+K \cdot\left[\begin{array}{cc}
\lambda_{1}^{t} & 0 \\
0 & \lambda_{2}{ }^{t}
\end{array}\right] \cdot K^{-1} \cdot\left(\begin{array}{c}
m_{0}-\hat{m} \\
\pi_{0}{ }^{e}-\hat{\pi}^{e}
\end{array}\right)
\end{array}
$$

The dynamic IS-LM system will be stable if şi and only if

$$
\left|\lambda_{1}\right|<1 \text { şi }\left|\lambda_{2}\right|<1
$$

In this case

$$
\left(\begin{array}{l}
m_{t} \\
\pi_{t}^{e}
\end{array}\right) \longrightarrow\left(\begin{array}{l}
\hat{m} \\
\hat{\pi}^{e}
\end{array}\right)
$$

It is to be checked if the solutions of equation (39) satisfy condition (46) The discriminant of equation (39) is:

$$
\Delta=\alpha\left[(\alpha-4 \delta)+\delta \cdot \alpha \cdot l_{2}\left(\delta \cdot l_{2}-2\right)\right]
$$

If $\delta>\frac{1}{4} \alpha$, then the discriminant will be negative, hence $\lambda_{1}$ and $\lambda_{2}$ will be complex conjugates.

In this case :

$$
\begin{aligned}
& \left|\lambda_{1}\right|=\left|\lambda_{2}\right|=\sqrt{1-\alpha+\delta \cdot \alpha+\delta \cdot \alpha \cdot l_{2}} \\
& \left|\lambda_{1}\right|<1 \text { if } \delta<\frac{1}{1+l_{2}}
\end{aligned}
$$

In conclusion, it follows that the stability of the dynamic IS-LM model depinds in a decisive way on the learning parameter $\delta$, which characterizes the manner in which economic agents form expectations of future inflation (relation $15)$.

If the learning parameter satisfies

$$
\frac{1}{4} \alpha<\delta<\frac{1}{1+l_{2}}
$$

i.e.:

$$
\frac{1}{4} \theta \cdot k \cdot \frac{g}{l_{2}}<\delta<\frac{1}{1+l_{2}}
$$

then the dynamic IS-LM system is stable. Relation (48) can be written as:

$$
\frac{1}{4} \theta \cdot \frac{\frac{g}{l_{2}}}{1-c+g \frac{l_{1}}{l_{2}}}<\delta<\frac{1}{1+l_{2}}
$$


We stress that relations (47), (48) and (49) represent sufficient conditions for stability, but not necessary conditions .

\section{$\underline{3 .}$ Phase portrait of the trajectories}

In order to represent graphically the dynamics of the system, we represent in the phase plane $\left(\pi^{e}, m\right)$ stationary trajectories (25) and (26).

The stationary trajectory for the real money supply is:

$$
\begin{aligned}
& \mathrm{m}_{\mathrm{t}+1}=m_{t}=m, \forall t \in \mathrm{N}, \text { or } \\
& m=-\frac{1+\alpha \cdot l_{2}}{\alpha} \pi^{e}+\frac{1}{\alpha}(\mu-h)
\end{aligned}
$$

The graph of the line (50) is represented in Figure 1.

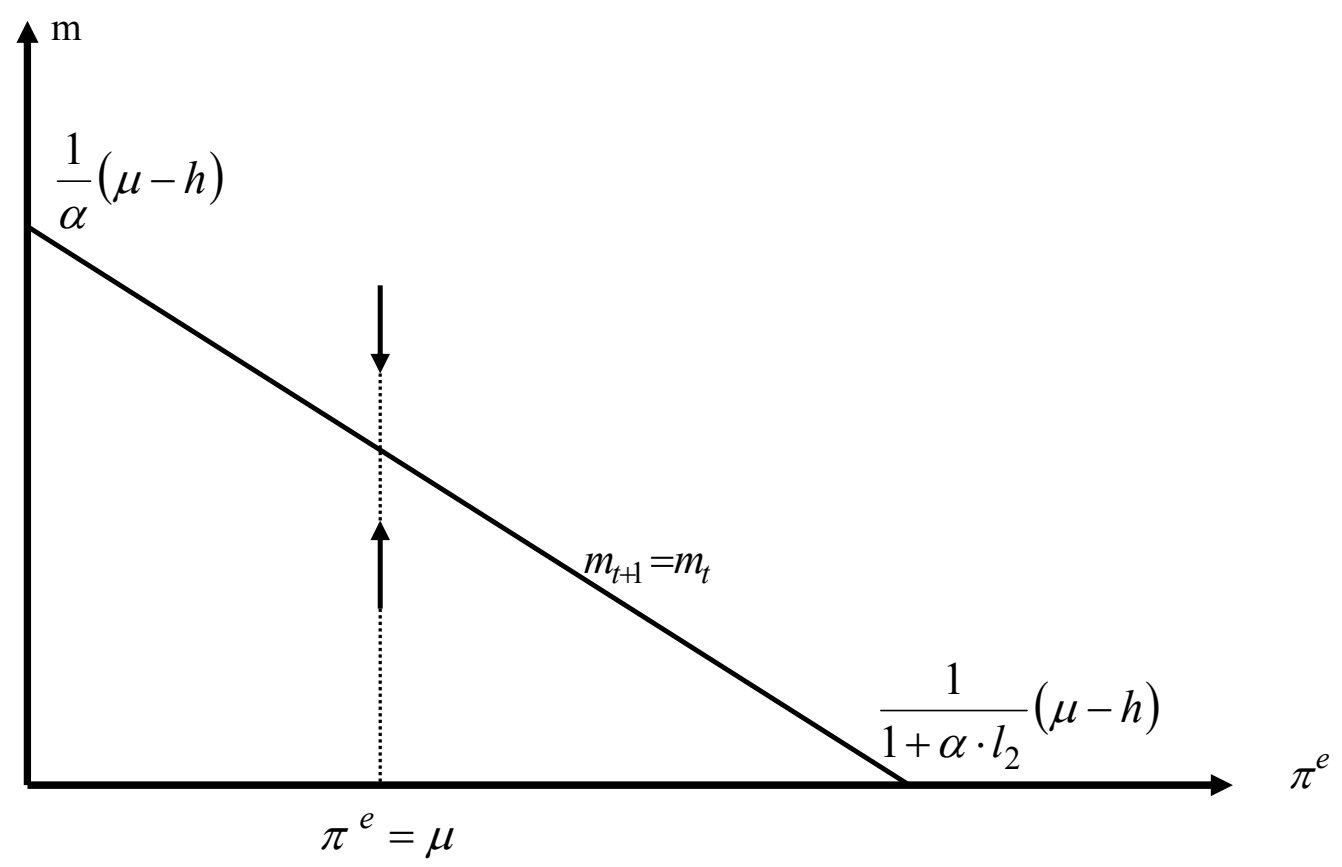

Figure 1

The line $m_{t+1}=m_{t}$ in Figure 1 divides the plane in two half-planes. For the points with coordnates $\left(\pi^{e}, m\right)$ in one of the half-planes $\mathrm{m}_{\mathrm{t}+1}>m_{t}$, and for the points in the other half-plane $\mathrm{m}_{\mathrm{t}+1}<m_{t}$.

In order to identify the sense of motion in each of the half-panes, we rewrite the first equation in (23) as :

$$
m_{t+1}-m_{t}=-\alpha\left(m_{t}+\frac{1+\alpha \cdot l_{2}}{\alpha} \pi_{t}^{e}-\frac{1}{\alpha}(\mu-h)\right)
$$


From the above relation it can be seen that, if

$$
m_{t}>-\frac{1+\alpha \cdot l_{2}}{\alpha} \pi_{t}^{e}+\frac{1}{\alpha}(\mu-h)
$$

then $\mathrm{m}_{\mathrm{t}+1}-m_{t}<0$

It follows that the sense of motion in the two half-planes is given by the arrows in Figure 1.

Analogously, we consider the line $\pi_{t+1}{ }^{e}=\pi_{t}{ }^{e}$, or

$$
m=-l_{2} \cdot \pi^{e}-\frac{1}{\alpha} h
$$

The graph of the stationary trajectory (51), as well as the sense of motion in the two half-planes defined by it are represented in Figure 2.

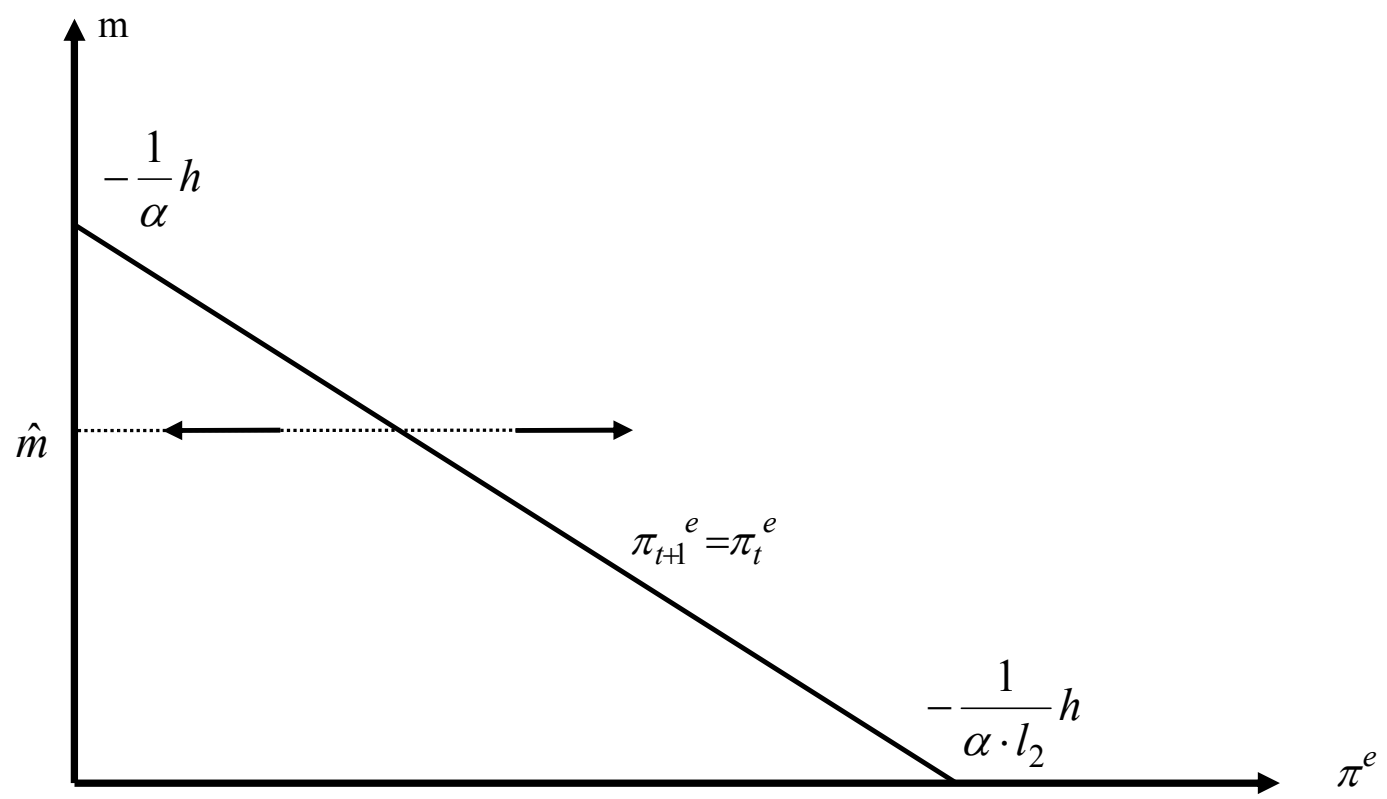

Figure 2

The sense of motion presented in Figure 2 results clearly from the second equation (23), which can be written as:

$$
\pi_{t+1}^{e}-\pi_{t}^{e}=\delta \cdot \alpha\left(m_{t}+l_{2} \cdot \pi_{t}^{e}+\frac{1}{\alpha} h\right)
$$

Representing both stationary trajectories in the same reference system, the phase space will be divided into four quadrants. The sense of motion in each of these quadrants is presented in Figure 3. 


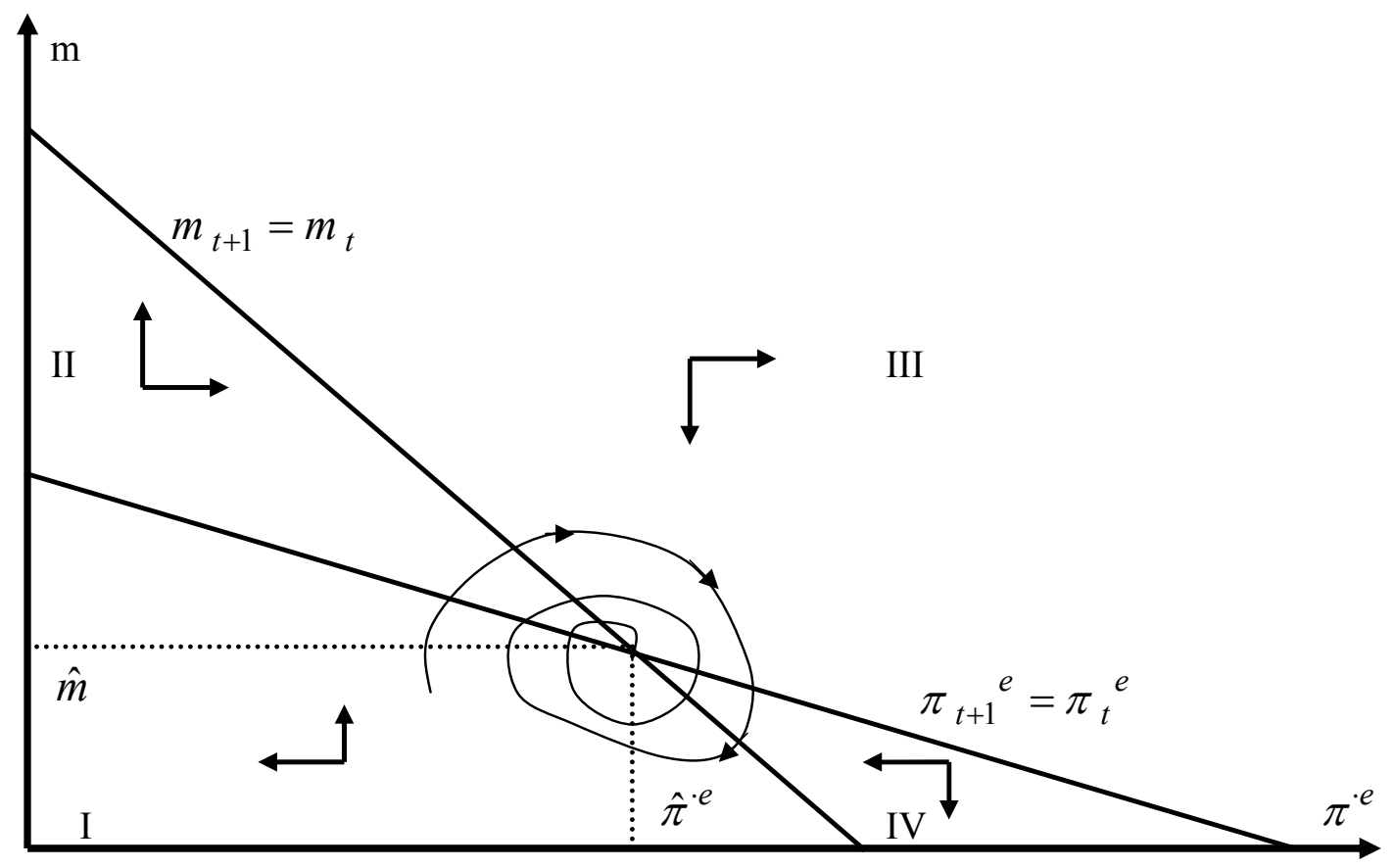

Figure 3 


\section{References}

Azariadis, C. (1993) Intertemporal Macroeconomics, Oxford, Blackwell.

Blanchard, O. and Fischer, S. (1989) Lectures on Macroeconomics, Massachusetts Institute of Technology Press.

Cagan, P. (1956) "The monetary Dynamics of Hyperinflation". In Studies in the Quantity Theory of Money, ed. M. Freedman, University of Chicago Press.

Casares, M. and Bennett Mc Callum (2000), "An Optimiying IS-LM Framework with Endogenous Investment”. NBER Working paper no. 7908.

De Cesare, L. and Mario Sportelli (2005) "A dynamic IS-LM model with delayed taxation revenue," Chaos, Solitons \& Fractals, 233-244, july 2005.

Henri, U. and B. Venturi (2007) "Stability and bifurcation in IS-LM economic models," International Review of Economics, vol. 54, no. 1, march 2007.

King, R. (2000) "The New IS-LM Model: Language, Logic and Limit" Federal Reserve Bank of Richmond Economic Quarterly.

Mc Callum, B. and Edward Nelson (2000) "An Optimizing IS-LM Specification for Monetary Policy and Business Cycle Analysis, " NBER working paper 5875 .

Romer, D. (2000) "Keynesian Macroeconomics without the LM Curve", NBER Working Paper 7461. 